\title{
Common nonlinear features and spin-orbit coupling effects in the Zeeman splitting of novel wurtzite materials
}

\author{
Paulo E. Faria Junior, ${ }^{1,}$ Davide Tedeschi, ${ }^{2}$ Marta De Luca, ${ }^{2,3}$ Benedikt Scharf, ${ }^{1,4}$ Antonio Polimeni, ${ }^{2}$ and Jaroslav Fabian ${ }^{1}$ \\ ${ }^{1}$ Institute for Theoretical Physics, University of Regensburg, 93040 Regensburg, Germany \\ ${ }^{2}$ Dipartimento di Fisica, Sapienza Università di Roma, 00185 Rome, Italy \\ ${ }^{3}$ Department of Physics, University of Basel, 4056 Basel, Switzerland \\ ${ }^{4}$ Institute for Theoretical Physics and Astrophysics, University of Würzburg, 97074 Würzburg, Germany
}

(Received 22 November 2018; published 22 May 2019)

\begin{abstract}
The response of semiconductor materials to external magnetic fields is a reliable approach to probe intrinsic electronic and spin-dependent properties. In this study, we investigate the common Zeeman splitting features of novel wurtzite materials, namely, InP, InAs, and GaAs. We present values for the effective $g$ factors of different energy bands and show that spin-orbit coupling effects, responsible for the spin splittings, also have noticeable contributions to the $g$ factors. Within the Landau level picture, we show that the nonlinear Zeeman splitting recently explained in magnetophotoluminescence experiments for InP nanowires by D. Tedeschi et al. [Phys. Rev. B 99, 161204 (2019)] is also present in InAs, GaAs, and even the conventional GaN. Such nonlinear features stem from the peculiar coupling of the A and B valence bands as a consequence of the interplay between the wurtzite crystal symmetry and the breaking of time-reversal symmetry by the external magnetic field. Moreover, we develop an analytical model to describe the experimental nonlinear Zeeman splitting and apply it to InP and GaAs data. Extrapolating our fitted results, we found that the Zeeman splitting of InP reaches a maximum value, which is a prediction that could be probed at higher magnetic fields.
\end{abstract}

DOI: 10.1103/PhysRevB.99.195205

\section{INTRODUCTION}

Novel III-V semiconductor compounds with wurtzite (WZ) crystal structure, such as InP [1-3], InAs [4,5], and GaAs [6,7], can nowadays be synthesized as nanowhiskers or nanowires (NWs) [8,9] with large diameters. In contrast to the widely studied zinc-blende (ZB) phase [10], the stablest crystal structure of non-nitride III-V compounds, there are still many unknown, or at least not completely understood, properties of these WZ materials, especially regarding spindependent phenomena [11]. For instance, spin-orbit coupling (SOC) parameters and effective $g$ factors in WZ NWs control the physics behind the exotic Majorana bound states in semiconductor/superconductor setups [12-15], spin-laser operation [16,17], and spin-relaxation mechanisms [18,19] and can be drastically modified under lateral quantum confinement $[20,21]$.

One of the possibilities to probe the intrinsic spin properties of a semiconductor system is to investigate their response under external magnetic fields, for instance, coupled to optical excitation in magnetophotoluminescence (magneto-PL) experiments. Recent studies investigated the Zeeman splitting (ZS) from magneto-PL $[1,7,22,23]$ and extracted effective $g$ factors using the conventional linear dispersion of the WZ ZS $[24,25]$. Despite the successful description of the ZS for the magnetic field oriented perpendicular to the NW axis (with a [0001] growth direction), this theoretical modeling has two main disadvantages for the magnetic field oriented parallel to

*fariajunior.pe@gmail.com the NW axis: (i) the effective $g$ factors of electrons and holes cannot be probed separately because of the optical transitions, and (ii) this theory does not account for the unconventional nonlinear ZS observed. Such nonlinear features have recently been observed in quantum dots [26,27] and quantum wells $[28,29]$, i.e., semiconductor systems with strong quantum confinement.

On the other hand, the case of WZ NWs is quite different since the NWs used in these recent experiments have a large diameter and effectively behave as a bulk material [3,30-32] with negligible lateral quantum confinement. Particularly, for InP WZ, it was unambiguously shown in the study of Tedeschi et al. [23] that these nonlinear features originate from the peculiar coupling of Landau levels (LLs) from different energy states in the valence band, specifically between A and B bands. Although this nonlinear ZS has also been observed in InGaAs [22] and GaAs [7], it remains to be shown that indeed these nonlinear features have the same origin and could be described in a compact analytical way. Furthermore, InAs WZ NWs have been investigated by recent transport experiments $[14,15,33,34]$. However, to the best of our knowledge, no theoretical attempt has ever been made to compute the effective $g$ factors in such a material.

In this paper, we analyze the ZS of novel III-V WZ materials, namely, InP, InAs, and GaAs. We provide the values for effective $g$ factors of different energy bands and highlight important contributions due to SOC effects originating from the interband SOC interaction. Turning to the LL physics, we apply the theoretical approach presented in the study of Tedeschi et al. [23] to show that the nonlinear ZS arises solely 
from the mixing within the valence band and it is indeed a common feature present in the studied materials. Based on this common mechanism responsible for the nonlinear features, we developed an analytical model that reliably fits the available experimental data, especially for InP WZ. We then extrapolate our fitted results and show that the nonlinear feature acts as a limiting effect to the maximum value of the ZS for InP. Under higher magnetic fields, such features could be observed experimentally in order to test the limits of our suggested model.

We organize this paper as follows: In Sec. II we discuss the effective $g$ factor calculations and the role of SOC effects. In Sec. III we show the common nonlinear features arising in the valence band from the LL coupling. The effective analytical model for the nonlinear ZS is presented in Sec. IV, and we draw our conclusions in Sec. V. In the Appendix, we discuss the LL spectra for ZB materials.

\section{EFFECTIVE $g$ FACTORS AND SPIN-ORBIT COUPLING EFFECTS}

In order to evaluate the effective $g$ factors within the $k \cdot p$ framework, we use the standard perturbative approach [35-37] that accounts for the coupling between different energy bands. Here we focus on the energy bands around the band gap at the $\Gamma$ point of WZ crystals, namely, the conduction band (CB) and the top three valence bands, labeled A, B, and C from highest to lowest energy. In Fig. 1(a) we depict the bulk WZ band structure and identify the labels for the different energy bands. Within this $k \cdot p$ perturbative approach, each band (twofold degenerate) is described by an effective Zeeman term of the form

$$
H_{\mathrm{ZS}}\left(B_{\alpha}\right)=\frac{\mu_{B}}{2} B_{\alpha} g_{\alpha} \tau_{\alpha}, \alpha=x, y, z,
$$

in which the matrices $\tau_{\alpha}$ are the Pauli matrices for the twofolddegenerate $\Gamma$-point states and the effective $g$ factor $g_{\alpha}$ is
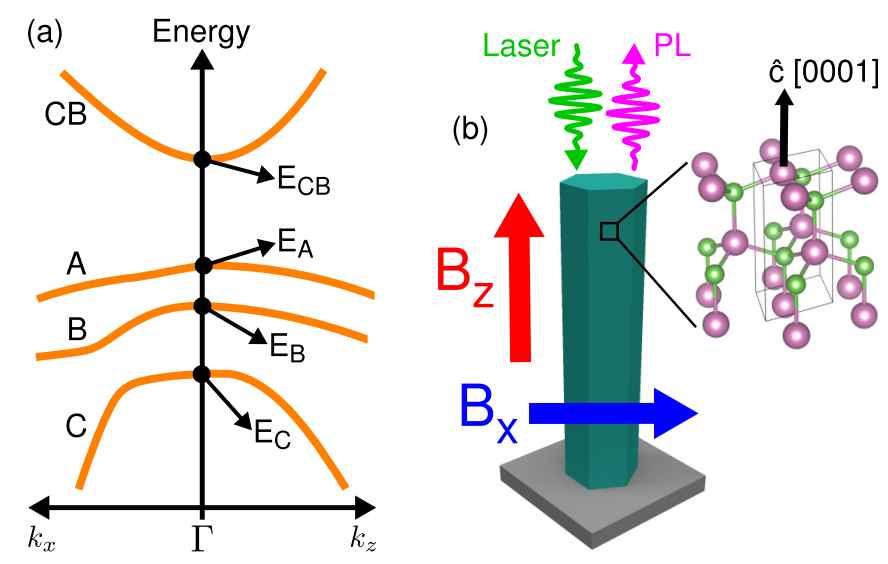

FIG. 1. (a) Schematic band structure for bulk WZ crystals around the $\Gamma$ point, indicating the different energy bands: $\mathrm{CB}, \mathrm{A}, \mathrm{B}$ and $\mathrm{C}$ (from top to bottom). The energies at the $\Gamma$ point are indicated by the arrows. (b) Scheme of the magnetic field configurations parallel $\left(B_{\mathrm{z}}\right)$ and perpendicular $\left(B_{\mathrm{x}}\right)$ to the NW axis in typical magneto-PL experiments. The laser excitation and the collected PL signal are parallel to the NW axis. The inset shows the WZ crystal structure with the orientation of the $c$ axis in the [0001] direction. obtained after evaluating

$$
g_{\alpha} \tau_{\alpha}^{n m}=g_{0} \sigma_{\alpha}^{n m}-i \frac{2 m_{0}}{\hbar^{2}} \sum_{l \neq n, m} \frac{\Pi_{\beta}^{n l} \Pi_{\gamma}^{l m}-\Pi_{\gamma}^{n l} \Pi_{\beta}^{l m}}{E_{n}-E_{l}},
$$

with $g_{0}\left(m_{0}\right)$ being the bare-electron $g$ factor (mass), $n, m$ being the states of a specific energy band at the $\Gamma$ point, $E_{n(l)}$ being the energy values at the $\Gamma$ point, and $\{\alpha, \beta, \gamma\}=$ $\{x, y, z\}$ (or cyclic permutations). The matrix elements of the Pauli matrices acting on the spin $1 / 2$ are given by $\sigma_{\alpha}^{a b}=$ $\left\langle a\left|\sigma_{\alpha}\right| b\right\rangle$, and the matrix elements for the $\vec{\Pi}$ operator are given by $\Pi_{\alpha}^{a b}=\left\langle a\left|\Pi_{\alpha}\right| b\right\rangle$, with the $\vec{\Pi}$ operator written as

$$
\vec{\Pi}=\frac{\hbar}{m_{0}} \vec{p}+\frac{\hbar^{2}}{4 m_{0}^{2} c^{2}}[\vec{\sigma} \times \vec{\nabla} V(\vec{r})]
$$

in which the second term describes the SOC contribution [38]. In Fig. 1(b) we show the direction of magnetic fields with respect to the WZ crystal structure.

To compute the $g$ factors in Eq. (2) we must specify a particular $k \cdot p$ Hamiltonian that contains the coupling among the different energy bands. For the $\mathrm{CB}, \mathrm{A}, \mathrm{B}$, and $\mathrm{C}$ bands including spin, the most general $8 \times 8 k \cdot p$ Hamiltonian that includes both orbital [first term in Eq. (3)] and SOC [second term in Eq. (3)] terms is given in Ref. [39]. It is convenient to notice that the matrix elements $\Pi_{\alpha}^{a b}$ can be easily obtained by looking at the Hamiltonian terms $H_{k p}^{(1)}$ and $H_{k S O}^{(1)}$ (shown in Appendix B of Ref. [39]). Furthermore, an additional parameter present in the Hamiltonian is the $k$-independent SOC $(\sim[\vec{\nabla} V(\vec{r}) \times \vec{p}] \cdot \vec{\sigma})$ between conduction and valence bands denoted by $\Delta_{4}$ (sometimes also called $\Delta_{s z}$ [40]). The inclusion of $\Delta_{4}$ prevents the analytical diagonalization of the Hamiltonian at the $\Gamma$ point.

The most straightforward way to calculate the $g$ factors is to evaluate Eq. (2) numerically, especially if the Hamiltonian does not allow analytical solutions. However, to unambiguously identify the contribution of SOC, we present here the analytical expressions for $\mathrm{CB}$ and $\mathrm{A}$ band $g$ factors assuming $\Delta_{4}=0$, which allows the analytical diagonalization of the WZ Hamiltonian at the $\Gamma$ point (see Sec. II B of Ref. [41], for instance). Rewriting the total $g$ factor of Eq. (2) as $g_{\alpha}=$ $g_{0}+\frac{2 m_{0}}{\hbar^{2}}\left(L_{\alpha}+\lambda_{\alpha}\right)$, we can identify the orbital contributions in $L_{\alpha}$ and the SOC effects in $\lambda_{\alpha}$. These terms for the CB and A bands read

$$
\begin{aligned}
& L_{x}^{\mathrm{CB}}= \sqrt{2} P_{1} P_{2} a b\left(\frac{1}{\Delta_{\mathrm{C}}}-\frac{1}{\Delta_{\mathrm{B}}}\right), \\
& \lambda_{x}^{\mathrm{CB}}= \frac{1}{\Delta_{\mathrm{B}}}\left[2 a \beta_{1}\left(a \beta_{1}-\sqrt{2} b \beta_{2}\right)\right. \\
&\left.+b P_{1}\left(2 b \beta_{2}-\sqrt{2} a \beta_{1}\right)+2 a^{2} \beta_{1} P_{2}\right] \\
&+\frac{1}{\Delta_{\mathrm{C}}}\left[2 b \beta_{1}\left(b \beta_{1}+\sqrt{2} a \beta_{2}\right)\right. \\
&\left.+a P_{1}\left(2 a \beta_{2}+\sqrt{2} b \beta_{1}\right)+2 b^{2} \beta_{1} P_{2}\right], \\
& L_{z}^{\mathrm{CB}}=P_{2}^{2}\left(\frac{b^{2}}{\Delta_{\mathrm{C}}}+\frac{a^{2}}{\Delta_{\mathrm{B}}}-\frac{1}{\Delta_{\mathrm{A}}}\right), \\
& \lambda_{z}^{\mathrm{CB}=} \frac{1}{\Delta_{\mathrm{A}}} \beta_{1}\left(2 P_{2}-\beta_{1}\right)
\end{aligned}
$$




$$
\begin{aligned}
& +\frac{1}{\Delta_{\mathrm{B}}}\left[\left(a \beta_{1}-\sqrt{2} b \beta_{2}\right)^{2}+2 a P_{2}\left(a \beta_{1}-\sqrt{2} b \beta_{2}\right)\right] \\
& +\frac{1}{\Delta_{\mathrm{C}}}\left[\left(b \beta_{1}+\sqrt{2} a \beta_{2}\right)^{2}+2 b P_{2}\left(b \beta_{1}+\sqrt{2} a \beta_{2}\right)\right],
\end{aligned}
$$

$$
\begin{aligned}
L_{z}^{A}= & -\frac{P_{2}^{2}}{\Delta_{\mathrm{A}}}+2 A_{7}^{2}\left(\frac{b^{2}}{\Delta_{\mathrm{AB}}}+\frac{a^{2}}{\Delta_{\mathrm{AC}}}\right), \\
\lambda_{z}^{A}= & -\frac{1}{\Delta_{\mathrm{A}}}\left(\beta_{1}^{2}-2 \beta_{1} P_{2}\right) \\
& +\frac{1}{\Delta_{\mathrm{AB}}}\left[\left(b \alpha_{1}+\sqrt{2} a \alpha_{2}\right)^{2}-2 b A_{7}\left(2 a \alpha_{2}+\sqrt{2} b \alpha_{1}\right)\right] \\
& +\frac{1}{\Delta_{\mathrm{AC}}}\left[\left(a \alpha_{1}-\sqrt{2} b \alpha_{2}\right)^{2}+2 a A_{7}\left(2 b \alpha_{2}-\sqrt{2} a \alpha_{1}\right)\right],
\end{aligned}
$$

with the energy differences given by $\Delta_{\mathrm{A}}=E_{\mathrm{CB}}-E_{\mathrm{A}}, \Delta_{\mathrm{B}}=$ $E_{\mathrm{CB}}-E_{\mathrm{B}}, \Delta_{\mathrm{C}}=E_{\mathrm{CB}}-E_{\mathrm{C}}, \Delta_{\mathrm{AB}}=E_{\mathrm{A}}-E_{\mathrm{B}}$, and $\Delta_{\mathrm{AC}}=$ $E_{\mathrm{A}}-E_{\mathrm{C}}$. The values $E_{\mathrm{CB}}, E_{\mathrm{A}}, E_{\mathrm{B}}$, and $E_{\mathrm{C}}$ are the energies at the $\Gamma$ point for the bands considered, indicated in Fig. 1(a). The $a$ and $b$ coefficients read $a=$ $\delta / \sqrt{\delta^{2}+2\left(\Delta_{3}\right)^{2}}$ and $b=\sqrt{2} \Delta_{3} / \sqrt{\delta^{2}+2\left(\Delta_{3}\right)^{2}}$, with $\delta=$ $\left(\Delta_{1}-\Delta_{2}\right) / 2+\sqrt{\left(\Delta_{1}-\Delta_{2}\right)^{2} / 4+2\left(\Delta_{3}\right)^{2}}$. The energy parameters $\Delta_{1}$ and $\Delta_{2,3}$ represent the crystal field splitting energy and the valence band SOC energies in WZ, respectively. The parameters $P_{1}$ and $P_{2}$ couple conduction and valence bands via the linear momentum operator, $A_{7}$ is the intravalence-band coupling also mediated by $\vec{p}, \beta_{1}$ and $\beta_{2}$ are SOC terms between conduction and valence bands, and $\alpha_{1}$ and $\alpha_{2}$ are SOC terms within only the valence band. For the precise definition of these couplings, please refer to Appendix B of Ref. [39].

The SOC corrections to the $g$ factors, shown in Eqs. (4)(6), take into account the same parameters that control the spin splitting of the energy bands (see, for instance, Eq. (10) in Ref. [39] for the CB spin-splitting parameters). Therefore, since the spin splittings of the energy bands are different (either in magnitude or $k$ dependence), so are the SOC corrections to the $g$ factors. We point out that $g_{x}^{\mathrm{A}}$ is not shown simply because it is zero due to the symmetry of the A bands (they do not couple via the $\Pi_{z}$ operator to any other band, and their different spin projections also do not couple by $\left.\sigma_{x}\right)$. Furthermore, we emphasize that by removing the SOC contribution (setting $\lambda_{\alpha}=0$ ) we recover the known result for the conduction band presented by Hermann and Weisbuch [36].

Now we turn to the calculated values of the effective $g$ factors for InP, InAs, and GaAs WZ. For InP and InAs we used the parameters (which contain SOC effects) available in Ref. [39], and for GaAs we used the parameters (without any SOC effects) from Ref. [42]. Please see Ref. [43] for more information on the $k \cdot p$ parameters used. We emphasize here that the SOC effects we refer to in the $k \cdot p$ Hamiltonians are related to the terms that contribute to the $g$ factor as shown in Eqs. (4)-(6) and the additional interband $k$-independent SOC term $\Delta_{4}$. Furthermore, in both $k \cdot p$ models $[39,42]$ the usual SOC in the valence band is included via the parameters $\Delta_{2}$
TABLE I. Calculated $g$ factors for the different energy bands of InP, InAs, and GaAs WZ. In our notation, the $z(x, y)$ direction is

\begin{tabular}{|c|c|c|c|c|c|}
\hline & \multirow[b]{2}{*}{ Band } & \multicolumn{2}{|c|}{ No SOC } & \multicolumn{2}{|c|}{ With SOC } \\
\hline & & $g_{x}$ & $g_{z}$ & $g_{x}$ & $g_{z}$ \\
\hline \multirow[t]{4}{*}{$\mathrm{InP}^{\mathrm{a}}$} & $C B$ & 1.72 & 1.81 & $1.29(25)$ & $1.61(11)$ \\
\hline & $A$ & 0.0 & -3.30 & 0.0 & $-3.05(8)$ \\
\hline & $B$ & -3.74 & 5.35 & $-3.94(5)$ & $5.12(4)$ \\
\hline & $C$ & 5.46 & 0.24 & $5.10(7)$ & $0.47(97)$ \\
\hline \multirow[t]{4}{*}{$\operatorname{In} A s^{a}$} & $C B$ & -5.49 & -5.33 & $-6.82(24)$ & $-6.23(17)$ \\
\hline & $A$ & 0.0 & -23.71 & 0.0 & -22.90 \\
\hline & $B$ & -19.69 & -10.81 & $-19.06(3.1)$ & $-8.97(17)$ \\
\hline & $C$ & 14.20 & 7.57 & $14.07(1)$ & $7.70(2)$ \\
\hline \multirow[t]{4}{*}{$\mathrm{GaAs}^{\mathrm{b}}$} & $C B$ & 0.33 & 0.46 & & \\
\hline & $A$ & 0.0 & -10.19 & & \\
\hline & $B$ & -9.17 & 7.22 & & \\
\hline & $C$ & 9.50 & -3.43 & & \\
\hline
\end{tabular}
parallel (perpendicular) to the $c$ axis of the WZ structure, as indicated in Fig. 1(b). The numbers in parentheses in the column "With SOC" indicate the approximate percentage of the SOC contribution to the $g$ factor, defined as $|[g(\mathrm{SOC})-g] / g|$, with $g$ being the $g$ factor without SOC effects.

${ }^{\text {a The } k \cdot p \text { parameters from Ref. [39]. }}$

${ }^{\mathrm{b}}$ The $k \cdot p$ parameters from Ref. [42].

and $\Delta_{3}$. In Table I we show the calculated $g$ factors in the absence of SOC and with SOC (if the $k \cdot p$ model allows). As a general trend, $g_{x}=g_{y} \neq g_{z}$ (highlighting the anisotropy of the WZ structure), and the valence bands have larger $g$ factors (in absolute value) than the CB. Taking into account the SOC effects, only available for InP and InAs, we notice that their correction to the $g$ factor values is, in general, not negligible and has values within the typical experimental precision. For instance, the influence of SOC can reach contributions of $\sim 25 \%$ in $\mathrm{CB} g$ factors, and it is larger for $g_{x}^{\mathrm{CB}}$ than $g_{z}^{\mathrm{CB}}$. The reason for this larger SOC effect in $g_{x}^{\mathrm{CB}}$ is due to the contribution of the parameter $P_{1}$ in $\lambda_{x}^{\mathrm{CB}}$ that is absent in $\lambda_{z}^{\mathrm{CB}}$ (and, additionally, $P_{1}>P_{2}$ ). It is also worth mentioning the $g$ factors for the $\mathrm{ZB}$ phase. Restricting ourselves to the $\mathrm{CB}$, the most commonly investigated case, the $\mathrm{ZB} g$ factor is $g^{*}=1.26$ for InP, $g^{*}=-14.9$ for InAs, and $g^{*}=-0.44$ for GaAs (with values taken from Ref. [44]). We notice that, indeed, the effective $g$ factor values are quite different for ZB and WZ crystal phases.

Let us now compare our theoretical $g$ factor values to experiments. In Table II we compare our calculated WZ $g$ factors with the available magneto-PL experimental data for InP and GaAs NWs (with diameters large enough to be considered a bulk system). Let us first discuss the InP case. For both $g_{x}^{\mathrm{CB}}$ and $g_{z}^{\mathrm{CB}}-g_{z}^{\mathrm{A}}$, our calculated values including the SOC contributions provide excellent agreement with the reported experimental values. We also point out the apparent inconsistency between the two experimental $g$ factors by showing the range of magnetic field used in the fitting. Since for magnetic fields along the $z$ direction the ZS is nonlinear, the larger the range used in the fitting is, the smaller the $g$ factors will be in order to account for the sublinear features. Therefore, we emphasize that experimentally determined $g$ factors should be 
TABLE II. Comparison between calculated and experimental values of the effective $g$ factors for InP and GaAs WZ.

\begin{tabular}{lccc}
\hline \hline & Source & $g_{x}^{\mathrm{CB}}$ & $g_{z}^{\mathrm{CB}}-g_{z}^{\mathrm{A}}$ \\
\hline InP & Expt. [23] & $1.3^{\mathrm{a}}$ & $4.4^{\mathrm{a}}$ \\
& Expt. [1] & $1.4^{\mathrm{b}}$ & $3.4^{\mathrm{b}}$ \\
& This work, with SOC & 1.29 & 4.66 \\
& This work, no SOC $^{\mathrm{c}}$ & 1.72 & 5.26 \\
GaAs & Expt. & $0.28^{\mathrm{d}}$ & $5.4^{\mathrm{e}}$ \\
& This work, no SOC & 0.33 & 10.83 \\
\hline \hline
\end{tabular}

${ }^{\mathrm{a}}$ Fitting up to $5 \mathrm{~T}$ (linear regime).

${ }^{\mathrm{b}}$ Fitting up to $15 \mathrm{~T}$ (already in nonlinear regime).

${ }^{\mathrm{c}}$ The $k \cdot p$ parameters from Ref. [39].

${ }^{\mathrm{d}}$ Reference [6], fitting up to $0.4 \mathrm{~T}$ (linear regime).

${ }^{\mathrm{e}}$ Reference [7], fitting up to $15 \mathrm{~T}$ (still linear regime).

${ }^{\mathrm{f}}$ The $k \cdot p$ parameters from Ref. [42].

fitted only at the limit of magnetic field values where the linear regime holds. For GaAs, although the comparison for $g_{x}^{\mathrm{CB}}$ looks reasonable, the theoretical value obtained for $g_{z}^{\mathrm{CB}}-g_{z}^{\mathrm{A}}$ is nearly twice as large as the experimental value. This clearly indicates that the $k \cdot p$ parameters for GaAs are not completely consistent and further theoretical efforts are required to build a more realistic model.

Although there is, to the best of our knowledge, no magneto-PL reported for pure InAs WZ NWs, it is important to mention that this material was recently investigated in several transport experiments $[14,15,33,34]$. Specifically, the conductance experiments by Vaitiekènas et al. [33] using 100 -nm InAs WZ NWs showed that for negative gate voltages the effective $g$ factor of $\mathrm{CB}$ electrons saturates to $\left|g^{*}\right| \sim 5$. This is significantly different from the bulk InAs ZB value of $g^{*}=-14.9$ but much closer to our predicted value for InAs WZ of $g_{z}^{\mathrm{CB}}=-6.23(-5.33)$ with (without) SOC effects. Furthermore, the recent theoretical analysis of ZB NWs by Winkler et al. [20] showed that orbital effects play a large role in the effective $g$ factors of different subbands. However, the lowest subband still retains much of the bulk information, especially at $100 \mathrm{~nm}$ (see, for instance, Figs. 2(c) and 2(d) in Ref. [20]). Although our theoretical value for the InAs WZ $g$ factor seems consistent with recent experiments and, more importantly, it is substantially different from the ZB value, further investigations that account for the electrostatic environment and quantum confinement in WZ NWs are still required, as pointed out in the conclusions of Ref. [33].

\section{NONLINEAR FEATURES IN LANDAU LEVELS}

Following the theoretical approach discussed in Ref. [23] to model the nonlinear ZS of InP WZ, we show in this section that indeed such nonlinear features are a common trend and also appear in the LLs of InAs and GaAs WZ. To calculate the LLs, we use the general description of an external magnetic field within the $k \cdot p$ framework which considers the envelope function approximation (EFA), combined with the minimal coupling and the Zeeman term $[37,45,46]$. The mathematical procedure of the EFA leads to the general Hamiltonian

$$
H=H_{\text {bulk }}\left[\vec{k} \rightarrow-i \vec{\nabla}+\frac{e}{\hbar} \vec{A}(\vec{r})\right]+g_{0} \frac{\mu_{B}}{2} \vec{\Sigma} \cdot \vec{B},
$$

in which $H_{\text {bulk }}(\vec{k})$ is the $k \cdot p$ bulk Hamiltonian, the replacement $\vec{k} \rightarrow-i \vec{\nabla}+\frac{e}{\hbar} \vec{A}(\vec{r})$ takes into account the EFA procedure, $g_{0}$ is the bare-electron $g$ factor, and $\vec{\Sigma}$ is a vector of the Pauli matrices describing the Zeeman term for the spins of the bulk Bloch functions $[45,46]$. By choosing the vector potential with a single spatial dependence, we can solve the Hamiltonian (7) numerically by introducing the finitedifference approach [47], similar to a quantum well treatment. The solution provides the LL spectra of the system, with energies denoted by $E_{\lambda}\left(k_{B}, k_{A}\right)$ and wave functions

$$
\psi_{\lambda, k_{B}, k_{A}}(\vec{r})=\frac{e^{i\left(k_{B} r_{B}+k_{A} r_{A}\right)}}{\sqrt{\Omega}} \sum_{l} f_{\lambda, k_{B}, k_{A}, l}(\rho) u_{l}(\vec{r}),
$$

in which $\lambda$ is the LL label, $f_{\lambda, k_{B}, k_{A}, l}(\rho)$ is the envelope function, the summation in $l$ runs over the bulk basis states denoted by $u_{l}(\vec{r}), \Omega$ is the area of the system perpendicular to the confinement direction, $k_{B}$ is the wave vector parallel to the magnetic field, $k_{A}$ is parallel to the vector potential, and the spatial dependence of the vector potential is denoted by the coordinate $\rho$. For the two directions of magnetic field investigated here [indicated in Fig. 1(b)] we have $\vec{B}=$ $B \hat{x} \Rightarrow \vec{A}=B y \hat{z}, k_{B}=k_{x}, k_{A}=k_{z}, \rho=y$ and $\vec{B}=B \hat{z} \Rightarrow \vec{A}=$ $B x \hat{y}, k_{B}=k_{z}, k_{A}=k_{y}, \rho=x$. To simplify the notation we set $\vec{B}=B \hat{x}$ as $B_{\mathrm{x}}$ and $\vec{B}=B \hat{z}$ as $B_{\mathrm{z}}$ in the remainder of the paper. We note that in experimental papers [1,7,22,23], $B_{\mathrm{X}}$ and $B_{\mathrm{Z}}$ are typically called the Voigt and Faraday configurations, respectively. For the numerical implementation of the Hamiltonian (7) we considered the system to have a size of $L=200 \mathrm{~nm}$ with 401 discretization points (with approximately 1 point every $0.5 \mathrm{~nm}$ ). For InP and InAs we used the bulk $8 \times 8 k \cdot p$ model from Ref. [39], and for GaAs we used the $6 \times 6 k \cdot p$ model from Ref. [42].

Due to the coupling of the A and B bulk energy bands induced by the external magnetic field, the valence band LLs show a markedly nonlinear behavior [23]. This common feature can be seen in Figs. 2(a)-2(c) for InP, InAs, and GaAs, respectively. We notice the clear nonlinear features in the different LLs whenever the B band mixing is present (indicated by the color code). Although for the $B_{\mathrm{x}}$ configuration this mixing is drastically reduced, partially because of the zero $g$ factor of the A band, the A-B mixing is indeed responsible for the slight nonzero ZS observed. As revealed in Ref. [23], the topmost LL (with branches indicated by $1+$ and $1-$ in Fig. 2) provides the main contribution to the excitonic effects, and therefore, the nonlinear features in the magneto-PL for the $B_{\mathrm{z}}$ configuration originate from the mixing of $\mathrm{A}$ and $\mathrm{B}$ valence bands. To highlight the nonlinear features, we show in Fig. 2(d) the ZS for the topmost LL in InP (solid line), InAs (long-dashed line), and GaAs (short-dashed line). We point out here that although the $6 \times 6$ Hamiltonian for GaAs WZ [42] is not sufficient to describe the linear Zeeman splitting correctly, since the $P_{1}$ and $P_{2}$ parameters are not included, the nonlinear features in the LLs are clearly visible. In fact, this is additional support for the fact that these nonlinear features are beyond the linear $g$ factor approach, which is mainly ruled by the $P_{1}$ and $P_{2}$ parameters, as shown in Eqs. (4)-(6).

In order to complete our analysis of the common nonlinear features of the valence band, we now discuss how the LL 


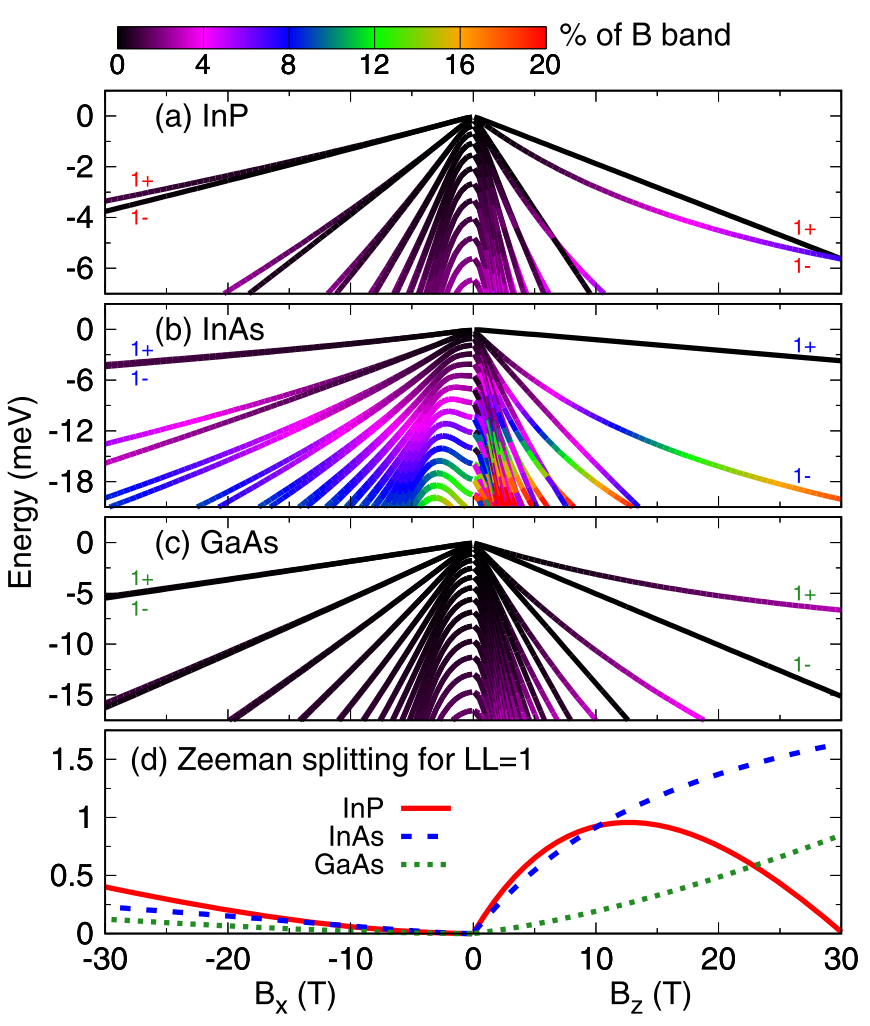

FIG. 2. Calculated Landau level spectra for the $\mathrm{A}$ band at $k_{B}=$ $k_{A}=0$ as a function of the magnetic field for (a) InP, (b) InAs, and (c) GaAs. The color code indicates the contribution of the $\mathrm{B}$ valence band to the total state. The upper (lower) branch of the topmost Landau level is indicated by $1+(1-)$. (d) Zeeman splitting for the topmost Landau level. We denote with positive (negative) values on the $x$ axis the magnetic field configuration $B_{\mathrm{z}}$ along the $z$ ( $B_{\mathrm{x}}$ along the $x$ ) direction.

coupling manifests in the envelope functions. We show in Fig. 3 the probability density of the envelope functions for the topmost LL (the 1+ and 1- branches shown in Fig. 2) in both magnetic field configurations at $30 \mathrm{~T}$. Due to the interplay of the WZ symmetry and the external magnetic field, the mixing of A and B bands has a peculiar form that couples envelope functions with different numbers of nodes, i.e., zero nodes for $\mathrm{A}$ bands and two nodes for B bands (further details on this coupling can be found in Ref. [23] and its Supplemental Material). In fact, due to strong SOC in InAs we also notice the contribution of $B$ states with one node [see Figs. 3(f) and 3(h)]. Moreover, for the $B_{\mathrm{z}}$ configuration the coupling between envelope functions is spin dependent, which means that the nonlinear feature is associated with one specific type of circular polarization (due to the conservation of angular momentum, spins in conduction and valence bands define the allowed transitions of circularly polarized light $[17,49])$. Indeed, this is exactly the case observed in recent magneto-PL experiments in InP WZ by Tedeschi et al. [23] that identified a strong nonlinear feature arising for a specific circular polarization of the PL spectra. On the other hand, for the $B_{\mathrm{x}}$ configuration, both spin components contribute equally, and as a consequence, the output light cannot be resolved in different circular polarizations.

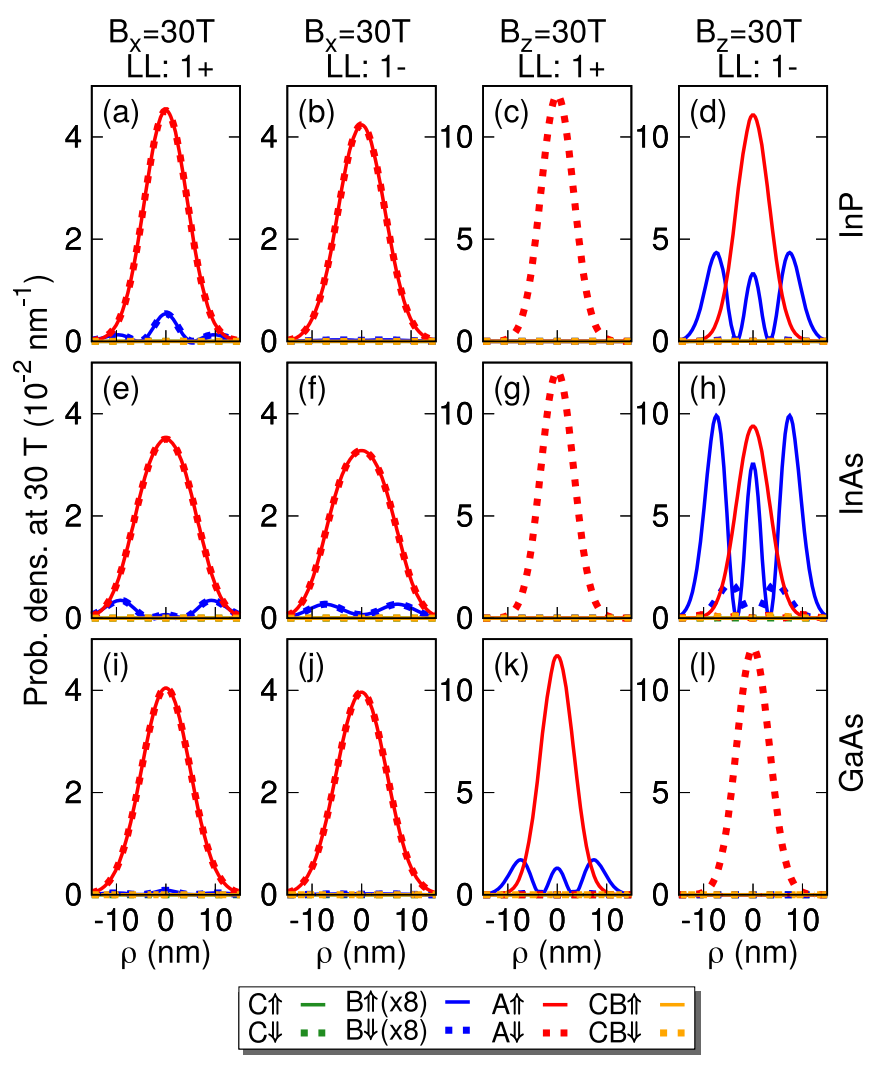

FIG. 3. Probability density of the envelope functions at $30 \mathrm{~T}$ for the first LL (indicated by the labels $1+$ and $1-$ in Fig. 2) along $B_{\mathrm{x}}$ and $B_{\mathrm{z}}$ for (a)-(d) InP, (e)-(h) InAs, and (i)-(l) GaAs. The B band envelope functions are multiplied by a factor of 8 , as indicated in the legend. The spin notation for the energy bands is identified with respect to the leading contribution of the bulk basis states [48].

\section{GaN wurtzite}

We discuss here the case of GaN, a well-established WZ compound in the family of nitrides that was recognized in the 2014 Nobel Prize in Physics for the efficient blue lightemitting diodes [50-52]. We focus on the LL spectra of the valence bands since a detailed discussion the effective $g$ factors in WZ GaN was performed by Rodina and Meyer [53]. In Fig. 4(a) we show the LL spectra for the A band of GaN. Due to the small SOC and crystal field energies of a few $\mathrm{meV}$ in $\mathrm{GaN}$, the mixing of $\mathrm{A}$ and $\mathrm{B}$ bands increases in comparison to the III-V WZ materials discussed above (notice the color scale in Figs. 2 and 4). For the ZS of the topmost LL, shown in Fig. 4(b), we notice that the nonlinear features are present for small values of magnetic field $(<5 \mathrm{~T})$, but the resulting ZS is $\sim 0.1 \mathrm{meV}$, which seems to be within the experimental error to be properly distinguished. Therefore, the overall behavior of the ZS can be modeled using a linear dispersion, as indicated in the experimental study of Rodina et al. [54]. Finally, we show in Figs. 4(c)-4(f) the probability densities for the envelope functions of the LL branches 1+ and $1-$ at $30 \mathrm{~T}$ for the magnetic field along $B_{\mathrm{x}}$ and $B_{\mathrm{z}}$ and find that the same coupling mechanisms as discussed for Fig. 3 take place. 

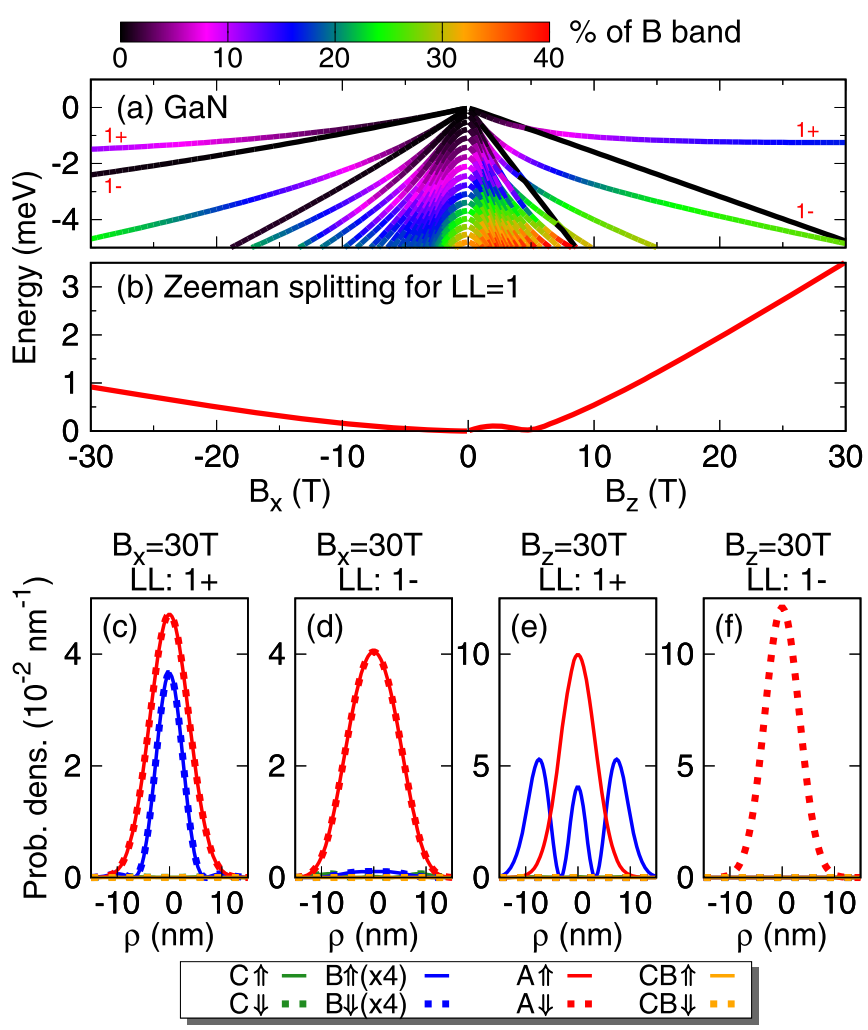

FIG. 4. (a) Calculated Landau level spectra for the A band at $k_{B}=k_{A}=0$ as a function of the magnetic field for GaN. (b) Zeeman splitting for the topmost Landau level. The color code and axis notation follow Fig. 2. (c)-(f) Probability density of the envelope functions at $30 \mathrm{~T}$ for the first LL, indicated by the labels $1+$ and $1-$ in (a), along $B_{\mathrm{x}}$ and $B_{\mathrm{z}}$. The $\mathrm{B}$ band envelope functions are multiplied by a factor of 4 , as indicated in the legend.

\section{EFFECTIVE MODEL FOR THE NONLINEAR ZEEMAN SPLITTING}

Based on the LL calculations, we showed that the physical mechanism behind the nonlinear $\mathrm{ZS}$ in the valence band is a common feature in WZ materials due to the mixing of $\mathrm{A}$ and $\mathrm{B}$ bands induced by magnetic field. It would be valuable to incorporate these features in an analytical expression that could be used to fit the experimental data beyond the linear ZS regime [24,25]. In order to capture the nonlinear effects present in the LL branch with spin up, we can restrict ourselves to the important contributions of the A-B mixing by using the basis set $\left\{f_{0, \mathrm{~A} \uparrow}|\mathrm{A} \uparrow\rangle, f_{2, \mathrm{~B} \Uparrow}|\mathrm{B} \uparrow\rangle\right\}$, in which the subindices 0 and 2 refer to the number of nodes in the envelope functions (see Fig. 3). We neglect here the minor contribution of the envelope function with one node since it appears only in InAs due to strong SOC. Therefore, for the coupling between the $\mathrm{A} \Uparrow$ and $\mathrm{B} \Uparrow \mathrm{LL}$ branches, we can write the following $2 \times 2$ Hamiltonian:

$$
H=\left[\begin{array}{cc}
0 & 0 \\
0 & E_{\mathrm{B}}
\end{array}\right]+\frac{\mu_{B}}{2} B\left[\begin{array}{cc}
g_{\mathrm{A}} & 0 \\
0 & g_{\mathrm{B}}
\end{array}\right]+B\left[\begin{array}{cc}
d_{\mathrm{A}} & d_{\mathrm{AB}} \\
d_{\mathrm{AB}} & d_{\mathrm{B}}
\end{array}\right],
$$

in which the first term indicates the energy separation between $A$ and $B$ valence bands in the bulk case (we set the energy of the A band to zero), the second term is the ZS due to the $g$ factor contribution, and the third term is the coupling
Hamiltonian that mixes $\mathrm{A}$ and $\mathrm{B}$ bands, which arises from the second-order $k \cdot p$ term $[39,41]$. Here we assume these couplings are parametrized by the variables $d_{\mathrm{A}}, d_{\mathrm{B}}$, and $d_{\mathrm{AB}}$. Diagonalizing the Hamiltonian (9), we find the energy for the A $\Uparrow$ branch as

$$
\begin{aligned}
E_{\mathrm{A} \Uparrow}(B)= & \frac{1}{2}\left[E_{\mathrm{B}}+\frac{\mu_{B}}{2} B g_{+}+d_{+} B\right. \\
& \left.+\sqrt{\left(E_{\mathrm{B}}-\frac{\mu_{B}}{2} B g_{-}-d_{-} B\right)^{2}+4 d_{\mathrm{AB}}^{2} B^{2}}\right],
\end{aligned}
$$

with $g_{ \pm}=g_{\mathrm{A}} \pm g_{\mathrm{B}}$ and $d_{ \pm}=d_{\mathrm{A}} \pm d_{\mathrm{B}}$. For the A $\Downarrow$ branch that does not couple to any other states, unlike $A \Uparrow$, we have simply the linear dependence in $B$, i.e.,

$$
E_{\mathrm{A} \Downarrow}(B)=-\frac{\mu_{B}}{2} B g_{\mathrm{A}}+B d_{\mathrm{A}},
$$

in which the first term is related to the $\mathrm{ZS}$ with opposite sign in the $g$ factor compared to the $\mathrm{A} \Uparrow$ branch and the second term is the energy shift of the LL branch with the same form as given in Eq. (9).

In order to model the $\mathrm{ZS}$ obtained from the experimental PL peaks, we must take into account not only the ZS of the valence but also of the conduction band since they are coupled via the optical transition. The total $\mathrm{ZS}$ can be written as the difference of the $\mathrm{ZS}$ of the conduction and valence bands, i.e., $\mathrm{ZS}_{\mathrm{CB}}-\mathrm{ZS}_{\mathrm{A}}[24,25,46]$. For the conduction band we can use the linear $g$ factor $\mathrm{ZS}, \mathrm{ZS}_{\mathrm{CB}}=\mu_{B} B g_{z}^{\mathrm{CB}}$, and for the A band we use $\mathrm{ZS}_{\mathrm{A}}=E_{\mathrm{A} \Uparrow}(B)-E_{\mathrm{A} \Downarrow}(B)$ [shown in Eqs. (10) and (11)]. Finally, the total $\mathrm{ZS}$ is given by

$$
\begin{aligned}
\mathrm{ZS}(B)= & \mu_{B} B\left(g_{z}^{\mathrm{CB}}-g_{z}^{\mathrm{A}}\right)-\frac{1}{2}\left[\left(E_{\mathrm{B}}-\frac{\mu_{B}}{2} B g_{-}-d_{-} B\right)\right. \\
& \left.+\sqrt{\left(E_{\mathrm{B}}-\frac{\mu_{B}}{2} B g_{-}-d_{-} B\right)^{2}+4 d_{\mathrm{AB}}^{2} B^{2}}\right],
\end{aligned}
$$

in which the unknown parameters are only $d_{-}$and $\left|d_{\mathrm{AB}}\right|$ if we assume the values for the effective $g$ factors and the energy separation of A and B bands given by theory or found experimentally by other means. We emphasize that if we set the coupling parameter $d_{\mathrm{AB}}$ to zero in Eq. (12), we recover the linear ZS already established in the literature by Refs. [24,25]. Furthermore, if we set the energy separation of A and B bands to zero $\left(E_{\mathrm{B}} \rightarrow 0\right)$, then all the terms in Eq. (12) become linear in the magnetic field, and the nonlinearities vanish. This condition would be equivalent to the case of $\mathrm{ZB}$ crystals that have degenerate heavy- and light-hole bands at the $\Gamma$ point and therefore would present a linear ZS (see, for instance, the experimental ZS of InP ZB in the Supplemental Material of Ref. [23]).

Applying the effective analytical ZS of Eq. (12) to the magneto-PL data of InP [23] and GaAs [7], we show in Fig. 5 that this model successfully captures the experimental trends, particularly for InP WZ. In the fitting, we assumed the $g$ factors and energy separations are known from theory and obtained the values for $d_{-}$and $\left|d_{\mathrm{AB}}\right|$ (given in the caption of Fig. 5). For GaAs we notice that the fitted parameters $d_{-}$and $\left|d_{\mathrm{AB}}\right|$ are nearly one order of magnitude larger than the values obtained for InP. We assign this feature to the overestimation of the GaAs $g$ factors in comparison with the 

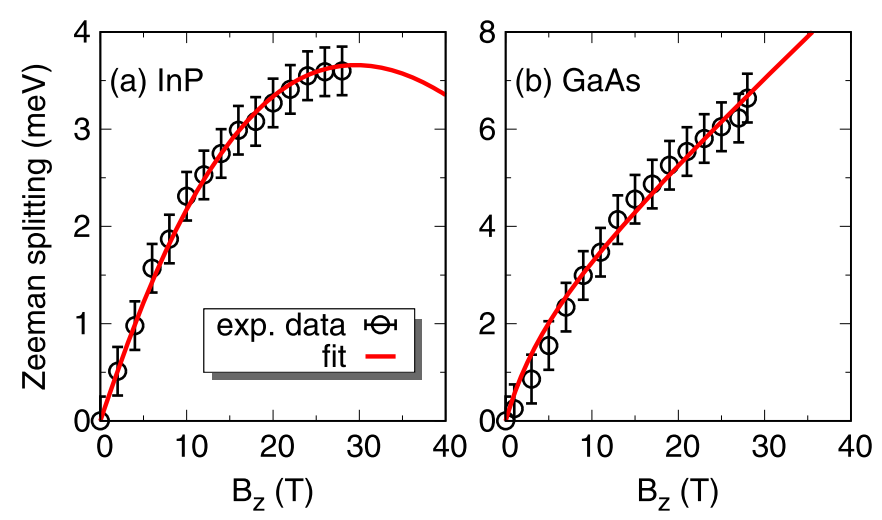

FIG. 5. Comparison of the fitting of Eq. (12) to the experimental Zeeman splitting for (a) InP and (b) GaAs. We use the calculated $g$ factors of Table I and the theoretical values of $E_{\mathrm{B}}=-35.4 \mathrm{meV}$ for InP [39] and $E_{\mathrm{B}}=-99.4 \mathrm{meV}$ for GaAs [42]. The fitting procedure provides $d_{-}=0.16 \mathrm{meV} / \mathrm{T},\left|d_{\mathrm{AB}}\right|=0.43 \mathrm{meV} / \mathrm{T}$ for InP and $d_{-}=$ $17.63 \mathrm{meV} / \mathrm{T},\left|d_{\mathrm{AB}}\right|=2.82 \mathrm{meV} / \mathrm{T}$ for GaAs. The experimental data for InP are taken from Ref. [23], and those for GaAs are from Ref. [7].

experimental data in the linear regime, shown in Table II. We emphasize that it is beyond the scope of this study to provide reliable interband couplings of GaAs WZ since additional theoretical efforts are required, such as $a b$ initio calculations with the correct conduction band ordering and a proper fitting of the $k \cdot p$ parameters, possibly including the SOC effects. Finally, we show that extrapolating our fitted curve up to $40 \mathrm{~T}$, we observe that the $\mathrm{ZS}$ of InP reaches a maximum value and then starts to decrease. This indicates that the nonlinear features act as a limiting factor to the maximum ZS that can be observed. For GaAs this feature is not visible due to the overestimated $g$ factors. Therefore, additional experimental data at magnetic fields higher than $30 \mathrm{~T}$ [55] could provide useful insight and also test the limits of the effective model presented in this study.

\section{CONCLUSIONS}

In summary, we theoretically investigated the common features of the Zeeman splitting in novel III-V wurtzite materials, namely, InP, InAs, and GaAs, using the $k \cdot p$ method. Specifically, we calculated the effective $g$ factors for the important energy bands around the band gap at the $\Gamma$ point $(\mathrm{CB}, \mathrm{A}, \mathrm{B}$, and $\mathrm{C}$ ) and showed that spin-orbit coupling effects have an appreciable contribution to the total $g$ factor values (contributing up to $\sim 20 \%$ of $\mathrm{CB}$, for instance). Our calculated values for the InP and InAs $g$ factors are in very good agreement with the available experimental values. Within the Landau level picture, following the prescription of Tedeschi et al. [23], we showed that the nonlinear Zeeman splitting for the $B_{\mathrm{z}}$ direction is a common feature of wurtzite materials due to the mixing of $\mathrm{A}$ and $\mathrm{B}$ valence bands induced by the external magnetic field. Relying on the main mechanism behind the origin of this nonlinear feature allowed us to develop an effective analytical description of the Zeeman splitting that describes the experimental data with very good agreement, particularly for InP WZ. By extrapolating our fitted model, we found that the nonlinear Zeeman splitting of InP WZ reaches a maximum value that could be investigated experimentally under magnetic fields higher than $30 \mathrm{~T}$. We also investigated the conventional wurtzite material $\mathrm{GaN}$ and showed that the nonlinear features are too weak to be visible experimentally. For zinc-blende materials, discussed in the Appendix, we showed that the valence band Zeeman splittings follow a strong linear behavior, especially for InP and GaAs.

Furthermore, our study shows that the $k \cdot p$ approach is very versatile, but it requires reliable parameter sets for quantitative comparison with the experimental data. For instance, the calculated $g$ factors we presented for GaAs do not provide a good description of the experimental data, indicating that further theoretical efforts in extracting reliable $k \cdot p$ parameters with the correct inclusion of SOC terms are needed. With the ongoing interest in these novel III-V WZ materials, with recent reports on high-quality samples of $\mathrm{GaP}$ [56] and $\mathrm{GaSb}$ [57], we believe our findings could guide future experiments and motivate further theoretical efforts to characterize these materials.
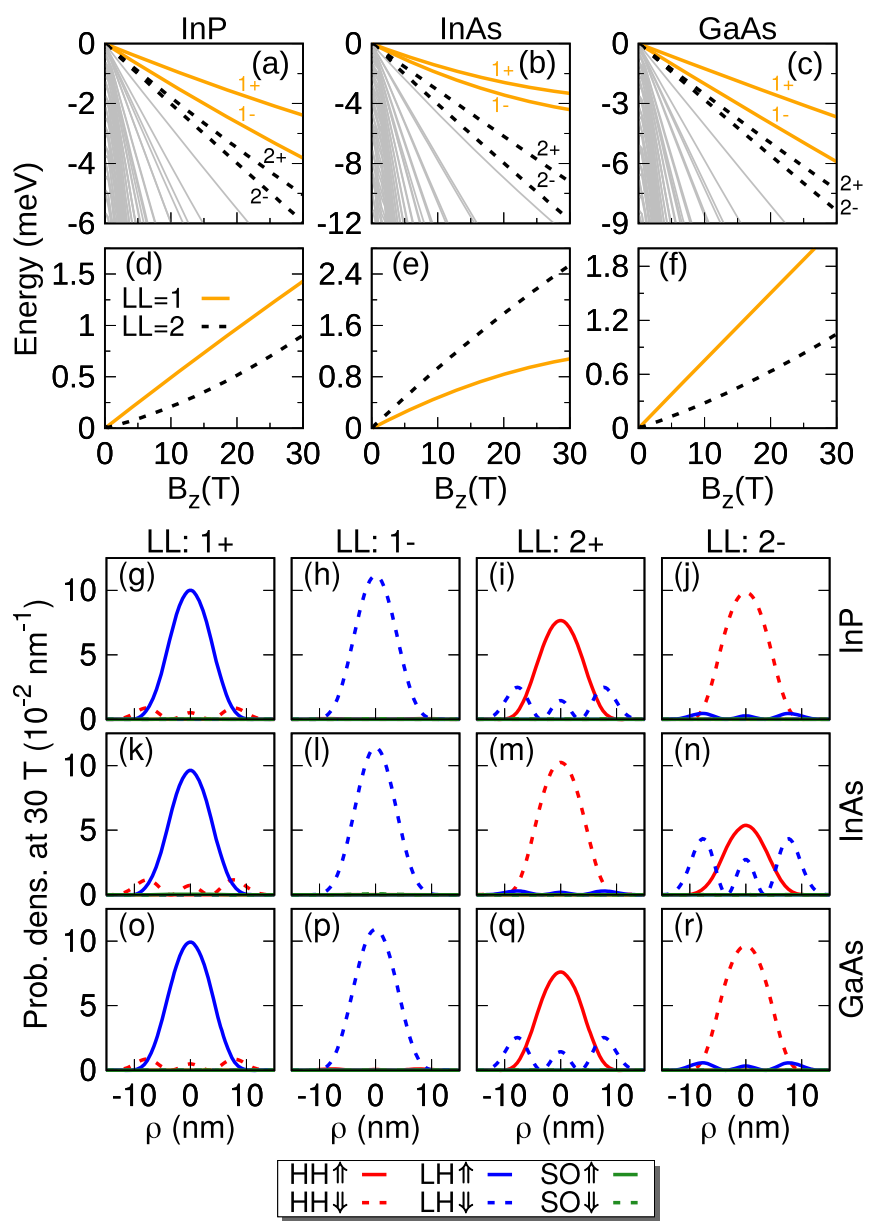

FIG. 6. Calculated Landau level spectra for a valence band at $k_{B}=k_{A}=0$ as a function of the magnetic field for (a) InP, (b) InAs, and (c) GaAs with a zinc-blende structure. The upper and lower branches of the first (thick solid lines) and second (thick dashed lines) topmost Landau levels are indicated by $1 \pm$ and $2 \pm$, respectively. Zeeman splitting for the first (solid lines) and second (dashed lines) topmost Landau levels for (d) InP, (e) InAs, and (f) GaAs. Probability densities at $30 \mathrm{~T}$ for $\mathrm{LL}=1$ and $\mathrm{LL}=2$ for (g)-(j) InP, (1)-(n) InAs, and (o)-(r) GaAs. 


\section{ACKNOWLEDGMENTS}

The authors acknowledge financial support from the Alexander von Humboldt Foundation, Capes (Grant No. 99999.000420/2016-06), SFB 1277 (B05), SFB 1170 "ToCoTronics," the ENB Graduate School on Topological Insulators, and Awards2014 and Avvio alla Ricerca (Sapienza Universitã di Roma). P.E.F.J. is grateful to D. R. Candido, T. Frank, J. Lee, and T. Campos for helpful discussions.

\section{APPENDIX: ZINC-BLENDE MATERIALS}

The LL formalism discussed in Sec. III can also be applied to $\mathrm{ZB}$ materials. Using the conventional Luttinger-Kohn $k \cdot p$ Hamiltonian [58] for the valence band combined with the Zeeman term [59], we calculate the LL spectra for InP, InAs, and $\mathrm{GaAs}$ with $\mathrm{ZB}$ crystal structure assuming a magnetic field along the [001] axis. The effective mass parameters are taken from Ref. [10], and the $\kappa$ parameters are taken from Ref. [44]. In Fig. 6 we present our calculations for the LL spectra, the
$\mathrm{ZS}$, and the probability densities focusing on the first and second topmost LL branches (denoted by $1 \pm$ and $2 \pm$ ), which have probability densities with a majority contribution of zero nodes. Specifically, in Figs. 6(a)-6(c) we show the LL spectra highlighting the $\mathrm{LL}=1$ branches (thick solid lines) and $\mathrm{LL}=$ 2 branches (thick dashed lines). In Figs. 6(d)-6(f) we show the $\mathrm{ZS}$ for $\mathrm{LL}=1$ and $\mathrm{LL}=2$ branches. Finally, in Figs. 6(g)-6(r) we show the probability densities at $B=30 \mathrm{~T}$. Although in these topmost LLs in ZB there is also mixing of the basis states for heavy- and light-hole bands (degenerate at the $\Gamma$ point), the $\mathrm{ZS}$ for the topmost LL is linear for InP and GaAs and slightly nonlinear for InAs, but not as pronounced as in WZ for the topmost LLs. A small nonlinear ZS can also be seen for $L L=2$. Finally, we point out that in typical magneto-PL experiments the topmost LL would be accessed via the optical transition, and therefore, the $\mathrm{ZS}$ for InP and GaAs would have just a linear dependence on magnetic field [please refer to Eq. (12) and the discussion below it for the case of degenerate bands with $E_{\mathrm{B}}=0$ ].
[1] M. De Luca, A. Polimeni, H. A. Fonseka, A. J. Meaney, P. C. M. Christianen, J. C. Maan, S. Paiman, H. H. Tan, F. Mura, C. Jagadish, and M. Capizzi, Nano Lett. 14, 4250 (2014).

[2] D. Tedeschi, M. De Luca, A. Granados del Águila, Q. Gao, G. Ambrosio, M. Capizzi, H. H. Tan, P. C. M. Christianen, C. Jagadish, and A. Polimeni, Nano Lett. 16, 6213 (2016).

[3] M. De Luca and A. Polimeni, Appl. Phys. Rev. 4, 041102 (2017)

[4] M. Möller, M. M. de Lima, Jr., A. Cantarero, T. Chiaramonte, M. A. Cotta, and F. Iikawa, Nanotechnology 23, 375704 (2012)

[5] M. B. Rota, A. S. Ameruddin, H. A. Fonseka, Q. Gao, F. Mura, A. Polimeni, A. Miriametro, H. H. Tan, C. Jagadish, and M. Capizzi, Nano Lett. 16, 5197 (2016).

[6] S. Furthmeier, F. Dirnberger, M. Gmitra, A. Bayer, M. Forsch, J. Hubmann, C. Schüller, E. Reiger, J. Fabian, T. Korn, and D. Bougeard, Nat. Commun. 7, 12413 (2016).

[7] M. De Luca, S. Rubini, M. Felici, A. Meaney, P. C. M. Christianen, F. Martelli, and A. Polimeni, Nano Lett. 17, 6540 (2017).

[8] V. Dubrovskii, G. Cirlin, and V. Ustinov, Semiconductors 43, 1539 (2009).

[9] P. Caroff, K. A. Dick, J. Johansson, M. E. Messing, K. Deppert, and L. Samuelson, Nat. Nanotechnol. 4, 50 (2009).

[10] I. Vurgaftman, J. R. Meyer, and L. R. Ram-Mohan, J. Appl. Phys. 89, 5815 (2001).

[11] I. Žutić, J. Fabian, and S. Das Sarma, Rev. Mod. Phys. 76, 323 (2004).

[12] R. M. Lutchyn, J. D. Sau, and S. Das Sarma, Phys. Rev. Lett. 105, 077001 (2010).

[13] Y. Oreg, G. Refael, and F. von Oppen, Phys. Rev. Lett. 105, 177002 (2010).

[14] A. Das, Y. Ronen, Y. Most, Y. Oreg, M. Heiblum, and H. Shtrikman, Nat. Phys. 8, 887 (2012).

[15] S. Albrecht, A. Higginbotham, M. Madsen, F. Kuemmeth, T. Jespersen, J. Nygård, P. Krogstrup, and C. Marcus, Nature (London) 531, 206 (2016).
[16] J.-Y. Chen, T.-M. Wong, C.-W. Chang, C.-Y. Dong, and Y.-F. Chen, Nat. Nanotechnol. 9, 845 (2014).

[17] P. E. Faria Junior, G. Xu, Y.-F. Chen, G. M. Sipahi, and I. Žutić, Phys. Rev. B 95, 115301 (2017).

[18] M. Kammermeier, P. Wenk, F. Dirnberger, D. Bougeard, and J. Schliemann, Phys. Rev. B 98, 035407 (2018).

[19] F. Dirnberger, M. Kammermeier, J. König, M. Forsch, P. E. Faria Junior, T. Campos, J. Fabian, J. Schliemann, C. Schüller, T. Korn, P. Wenk, and D. Bougeard, Appl. Phys. Lett. 114, 202101 (2019).

[20] G. W. Winkler, D. Varjas, R. Skolasinski, A. A. Soluyanov, M Troyer, and M. Wimmer, Phys. Rev. Lett. 119, 037701 (2017).

[21] T. Campos, P. E. Faria Junior, M. Gmitra, G. M. Sipahi, and J. Fabian, Phys. Rev. B 97, 245402 (2018).

[22] M. De Luca, A. Polimeni, M. Capizzi, A. J. Meaney, P. C. M. Christianen, J. K. Maan, F. Mura, S. Rubini, and F. Martelli, ACS Nano 7, 10717 (2013).

[23] D. Tedeschi, M. De Luca, P. E. Faria Junior, A. Granados del Águila, Q. Gao, H. H. Tan, B. Scharf, P. C. M. Christianen, C. Jagadish, J. Fabian, and A. Polimeni, Phys. Rev. B 99, 161204(R) (2019).

[24] K. Cho, Phys. Rev. B 14, 4463 (1976).

[25] H. Venghaus, S. Suga, and K. Cho, Phys. Rev. B 16, 4419 (1977).

[26] V. Jovanov, T. Eissfeller, S. Kapfinger, E. C. Clark, F. Klotz, M. Bichler, J. G. Keizer, P. M. Koenraad, M. S. Brandt, G. Abstreiter, and J. J. Finley, Phys. Rev. B 85, 165433 (2012).

[27] D. Y. Oberli, Phys. Rev. B 85, 155305 (2012).

[28] R. Kotlyar, T. L. Reinecke, M. Bayer, and A. Forchel, Phys. Rev. B 63, 085310 (2001).

[29] M. V. Durnev, M. M. Glazov, and E. L. Ivchenko, Phys. E (Amsterdam, Neth.) 44, 797 (2012).

[30] A. Mishra, L. V. Titova, T. B. Hoang, H. E. Jackson, L. M. Smith, J. M. Yarrison-Rice, Y. Kim, H. J. Joyce, Q. Gao, H. H. Tan, and C. Jagadish, Appl. Phys. Lett. 91, 263104 (2007).

[31] P. E. Faria Junior and G. M. Sipahi, J. Appl. Phys. 112, 103716 (2012). 
[32] L. C. O. Dacal and A. Cantarero, Sci. Rep. 6, 33914 (2016).

[33] S. Vaitiekėnas, M. T. Deng, J. Nygård, P. Krogstrup, and C. M. Marcus, Phys. Rev. Lett. 121, 037703 (2018).

[34] A. Iorio, M. Rocci, L. Bours, M. Carrega, V. Zannier, L. Sorba, S. Roddaro, F. Giazotto, and E. Strambini, Nano Lett. 19, 652 (2019).

[35] L. M. Roth, B. Lax, and S. Zwerdling, Phys. Rev. 114, 90 (1959).

[36] C. Hermann and C. Weisbuch, Phys. Rev. B 15, 823 (1977).

[37] L. C. Lew Yan Voon and M. Willatzen, The $k \cdot p$ Method: Electronic Properties of Semiconductors (Springer, Berlin, 2009).

[38] F. Dominguez, B. Scharf, G. Li, J. Schäfer, R. Claessen, W. Hanke, R. Thomale, and E. M. Hankiewicz, Phys. Rev. B 98, 161407(R) (2018). In the topological insulator bismuthene on $\mathrm{SiC}$, the interplay between such an increased effective $g$-factor and strong Rashba-like SOC gives rise to a sizable gap opened between counter propagating quantum spin Hall edge states by out-of-plane magnetic fields.

[39] P. E. Faria Junior, T. Campos, C. M. O. Bastos, M. Gmitra, J. Fabian, and G. M. Sipahi, Phys. Rev. B 93, 235204 (2016).

[40] J. Y. Fu and M. W. Wu, J. Appl. Phys. 104, 093712 (2008).

[41] S. L. Chuang and C. S. Chang, Phys. Rev. B 54, 2491 (1996).

[42] T. Cheiwchanchamnangij and W. R. Lambrecht, Phys. Rev. B 84, 035203 (2011).

[43] For InP and InAs WZ, the $k \cdot p$ parameters used from Ref. [39] were obtained by a numerical fitting to ab initio calculations performed within the density functional theory framework using the modified Becke-Johnson exchange potential with the local density approximation correlation. For GaAs WZ, the $k \cdot p$ parameters used from Ref. [42] are available only for the valence band and were obtained by a numerical fitting to $a b$ initio calculations performed within the quasiparticle selfconsistent GW method. For GaN WZ and III-V ZB materials we used the $k \cdot p$ parameters from Ref. [10], which were obtained by combining experimental and theoretical sources.

[44] R. Winkler, Spin-Orbit Coupling Effects in Two-Dimensional Electron and Hole Systems (Springer, New York, 2003).

[45] C. E. Pryor and M. E. Flatté, Phys. Rev. Lett. 96, 026804 (2006).
[46] J. van Bree, A. Yu. Silov, P. M. Koenraad, M. E. Flatté, and C. E. Pryor, Phys. Rev. B 85, 165323 (2012).

[47] S. L. Chuang and C. S. Chang, Semicond. Sci. Technol. 12, 252 (1997).

[48] Here we denote the bulk basis states as $\left\{\left|c_{1}\right\rangle, \ldots,\left|c_{8}\right\rangle\right\}$, representing the Bloch functions at the $\Gamma$ point without SOC interaction. From these eight states, states 1-6 represent the valence band, and states 7 and 8 represent the conduction band. Regarding spin- $1 / 2$ projections, states $1,2,3$, and 7 have spin up, while states $4,5,6$, and 8 have spin down. The bulk energy bands with SOC (the so-called $\mathrm{CB}, \mathrm{A}, \mathrm{B}$, and $\mathrm{C}$ ) are now described as a mixing of the eight basis states. Here we use the following convention: $\mathrm{CB} \Uparrow(\Downarrow)$ refers to the contribution of $c_{7}\left(c_{8}\right), \mathrm{A} \Uparrow(\Downarrow)$ refers to the contribution of $c_{1}\left(c_{4}\right), \mathrm{B} \Uparrow(\Downarrow)$ refers to the contribution of $c_{2}\left(c_{5}\right)$, and $\mathrm{C} \Uparrow(\Downarrow)$ refers to the contribution of $c_{3}\left(c_{6}\right)$. For the details of the basis states, please refer to Refs. [39,41].

[49] P. E. Faria Junior, G. Xu, J. Lee, N. C. Gerhardt, G. M. Sipahi, and I. Žutić, Phys. Rev. B 92, 075311 (2015).

[50] I. Akasaki, Rev. Mod. Phys. 87, 1119 (2015).

[51] H. Amano, Rev. Mod. Phys. 87, 1133 (2015).

[52] S. Nakamura, Rev. Mod. Phys. 87, 1139 (2015).

[53] A. V. Rodina and B. K. Meyer, Phys. Rev. B 64, 245209 (2001).

[54] A. V. Rodina, M. Dietrich, A. Goldner, L. Eckey, A. Hoffmann, Al. L. Efros, M. Rosen, and B. K. Meyer, Phys. Rev. B 64, 115204 (2001).

[55] Recent magneto-optics experiments in monolayer $\mathrm{WSe}_{2}$ reported magnetic field values up to $65 \mathrm{~T}$. A. V. Stier, N. P. Wilson, K. A. Velizhanin, J. Kono, X. Xu, and S. A. Crooker, Phys. Rev. Lett. 120, 057405 (2018).

[56] N. N. Halder, S. Cohen, D. Gershoni, and D. Ritter, Appl. Phys. Lett. 112, 133107 (2018).

[57] L. Namazi, L. Gren, M. Nilsson, M. Garbrecht, C. Thelander, R. R. Zamani, and K. A. Dick, Adv. Funct. Mater. 28, 1800512 (2018).

[58] J. M. Luttinger and W. Kohn, Phys. Rev. 97, 869 (1955).

[59] E. G. Novik, A. Pfeuffer-Jeschke, T. Jungwirth, V. Latussek, C. R. Becker, G. Landwehr, H. Buhmann, and L. W. Molenkamp, Phys. Rev. B 72, 035321 (2005). 